\title{
Commissioning and the future of diabetes care
}

\author{
VIJAY JAYAGOPAL ${ }^{1}$, REBECCA FIELD², TARA KADIS¹, JORDAN MCKIE', JOHN HAIGH², \\ ANDREW BUCKLEE², JONATHAN THOW ${ }^{1}$
}

\begin{abstract}
The creation of clinical commissioning groups has provided a unique opportunity for diabetes specialist teams and other stakeholders to collaborate directly with local commissioners to rationalise and improve diabetes care provision. We describe here the process by which the diabetes service in the Vale of York has been redesigned and a community diabetes service has been commissioned to help embed the new model of care. The process has involved getting key personnel in place to identify and drive change, creation of a model of care that is simple, removes artificial barriers and is based on clinical need rather than financial structures. Engaging and listening to stakeholders, particularly patients, was critical to the development as was participation of influential board level representatives to act as champions. Facilitative negotiation of the content and provision of the service helped build the business model to complement the clinical model. Developing a unified brand and image that would associate both commissioner and provider helped cement the alliance and provide patients with a single needs-based service.
\end{abstract}

Br J Diabetes Vasc Dis 2014;14:112-115

Key words: commissioning, diabetes, care model, community diabetes team

\section{Introduction}

Diabetes care is one of the major challenges facing the NHS. Direct costs account for an estimated $10 \%$ of total NHS expenditure and this is set to rise significantly. ${ }^{1}$ Of all the long term conditions, diabetes has the fastest rising prevalence. ${ }^{2,3}$ Current diabetes services are not considered value for money ${ }^{4}$ but, with improved services and management, it is estimated that savings

Centre for Diabetes and Endocrinology, York Teaching Hospital Foundation Trust, York, UK

Diabetes Redesign Project Team, Vale of York Clinical Commissioning Group, York, UK

Address for correspondence: Dr Vijay Jayagopal

Centre for Diabetes and Endocrinology, York Teaching Hospital

Foundation Trust, York, UK

Tel: +44 (0)1904 721175

E-mail: Vijay.Jayagopal@York.NHS.UK

http://dx.doi.org/10.15277/bjdvd.2014.021

\begin{tabular}{ll}
\multicolumn{2}{l}{ Abbreviations and acronyms } \\
CCG & Clinical Commissioning Group \\
CDT & Community diabetes team \\
CCG-DT & CCG-diabetes team \\
CSU & Commissioning Support Unit \\
DSN & diabetes specialist nurse \\
HDT & hospital diabetes team \\
IT & information technology \\
LETB & local education and training boards \\
NHS & National Health Service \\
PCT & Primary Care Trust \\
UoY & University of York \\
VoY- CCG & Vale of York Clinical Commissioning Group \\
YTHFT & York Teaching Hospital Foundation Trust
\end{tabular}

of up to $f 170$ million per year will be possible. ${ }^{3,4}$ Given the size of the diabetes population and that change can, in terms of outcomes, take decades to manifest, it is no surprise that diabetes service redesign is often overlooked for quicker gains.

The advent of CCG-based commissioning provides an opportunity to redress the historical malaise for change. Guidelines can help CCGs to use commissioning to promote large, meaningful service redesign..$^{5,6}$ It remains, however, a daunting task and for most clinical teams a nebulous concept. Despite the barriers, in January 2014 VoY-CCG agreed to commission a new community diabetes service. This has been achieved when CCG commissioning was in its infancy and working against a backdrop of financial constraints.

For the last year staff from VoY-CCG, the CSU and YTHFT, along with patient representatives, have worked together to redesign diabetes services for the population served by VoY-CCG. Reflecting on the journey, we feel confident that our process could act as a blueprint for how commissioning can drive service redesign. In this article we describe the circumstances that led to the need for change and the approaches taken to achieve our collective goal.

\section{Background}

Diabetes care in the Vale of York had evolved into a predominantly hospital-centric service, particularly for anyone requiring injectable therapies. Practice-based diabetes clinics varied considerably in care provision, with some caring for those with complex needs and others referring all insulin treated patients to hospital clinics. The local geography and relatively close proximity of some practices to the hospital may have influenced the development of this dichotomy. 
This service model did not suit the current national imperative of care closer to people's homes and the shift of services away from hospital to community-based models. Payment by results also created a barrier to integrated care for people with chronic disease. Discussions had commenced between the hospital team and the GP lead for diabetes, but progress was hampered by the disbanding of the local PCT and transition to the new CCG structure in April 2013.

The CCG executive introduced a hospital contract requiring a radical change in the new patient:follow-up ratio. In response to this, the records of all patients receiving hospital outpatient follow-up were reviewed to identify those who could be discharged to primary care. To facilitate this process the HDT agreed criteria for requiring hospital follow-up. About 1000 patients did not meet these criteria and were discharged back to their primary care teams. This created a fair degree of anxiety for patients and primary care teams which required an urgent solution.

\section{Critical appointment}

The HDT and patient groups highlighted the particular problem of diabetes care provision to the CCG executive who, to their credit, recognised the need for urgent review. A diabetes project manager and GP lead were appointed by the CCG to help review and re-organise services and the new liaison between the CCGDT and the HDT culminated in the commissioning of the new service.

\section{Focus of what we were trying to achieve}

Initial meetings helped baseline information collection, exchange of ideas, establishment of the need for change and the collective will to collaborate to achieve change. The strategic aim was to improve patient care by adopting a model of care that would be simple, free of artificial barriers and, most importantly, would base care on clinical need rather than financial structures.

Any hurdles or financial barriers were viewed as obstacles to be overcome. However any money saved by reducing hospital follow-up activity would be lost from diabetes care overall, thus it was imperative to find a mechanism that would ring fence finances for diabetes care.

The HDT offered to help design the new service model whilst being willing to work with whichever model the CCG-DT chose to commission. Listening and adopting a facilitative negotiating style helped smooth the discussions. This initial co-operation and openness set the tone for subsequent developments.

Figure 1. Model of care: To deliver integrated care, continuous quality improvement and to empower people in the management of their diabetes.

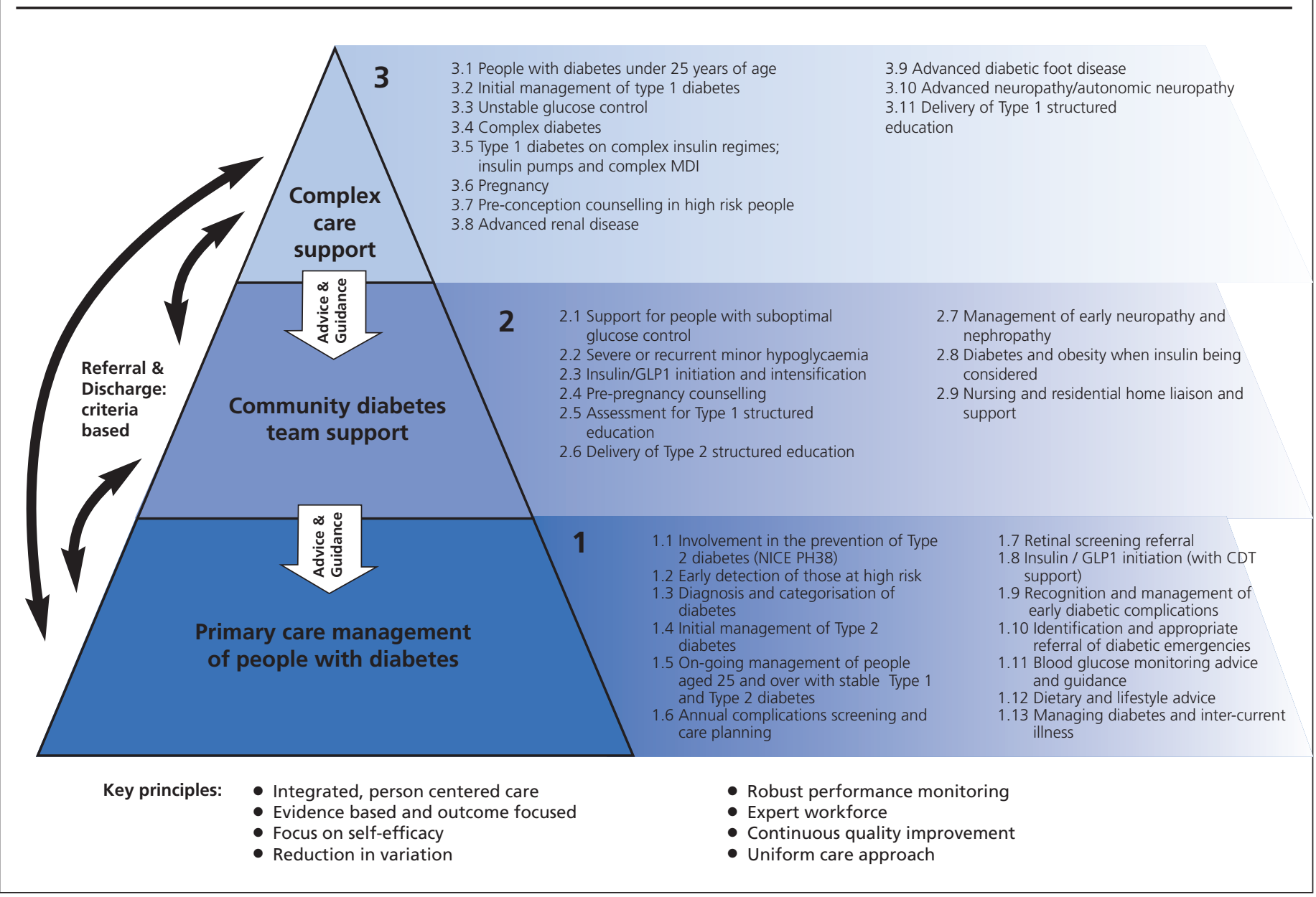




\section{Choosing a model}

The next phase involved the CCG-DT reviewing a series of care models (eg 'super 6', Joint ownership models, ${ }^{8}$ large integrated hospital-community based services ${ }^{9}$ ), and contacting and visiting centres with operational models. These activities informed a joint review and it was decided to create a bespoke model for York. The team created a model comprising three primary levels of care (Figure 1). This was accompanied by a list of activities that detailed the care that would be delivered at each level (Figure 1). When integrated into the IT system the links to referral forms, formulary and care pathways will be built in for added functionality.

The most important concept, hitherto unavailable in the region, was the creation of a CDT with a remit to support and underpin primary care teams to deliver high quality, equitable diabetes care. Additional roles included patient education to promote self care, governance support, practice benchmarking activities and providing needs-based short spells of direct patient support.

\section{Expanding the partnership}

It was recognised early in the process that successful delivery of the model required primary care teams to be confident about providing the expected level of activity. It was acknowledged that many centres would welcome upskilling and that would require readily accessible diabetes training. Following an initial scoping exercise involving local GPs and practice nurses, the HDT and UoY health sciences staff designed a Diabetes Masterclass module. The sessions were financed by existing LETB funding received by the UoY. This training programme would be backed up by online resources via the Which? YourDiabetes? website (more on this later).

Parallel to this, a project steering group was established which included patient representatives, a representative from Diabetes UK, the Directorate manager for medicine at YTHFT and a member of the CCG executive board. It was important to have key board level and patient champions onside to drive the process forward, but in hindsight greater representation from the contracting teams from the hospital and CCG would have saved us considerable time later on.

\section{Project plan and work streams}

Whilst the model was being discussed, the project lead helped create several work streams (Figure 2) involving GPs, practice nurses, DSNs, diabetologists, pharmacists, management staff, IT staff, UoY and patients.

The CCG and hospital contributed equal numbers in terms of staff input. Some of the CCG staff were contracted from the area CSU. The developments from these work streams helped refine the detail of the service and identify a total saving of about $£ 400,000$. A significant proportion of this was from accessing, validating and rationalising self monitoring of blood glucose. This involved a review of the choice and availability of testing equipment. Additional saving was identified from formulary changes and projected reduction in hospital based activity. A core operational group (CCG project manager, innovations manager, GP diabetes lead, DSN and
Figure 2. Work streams

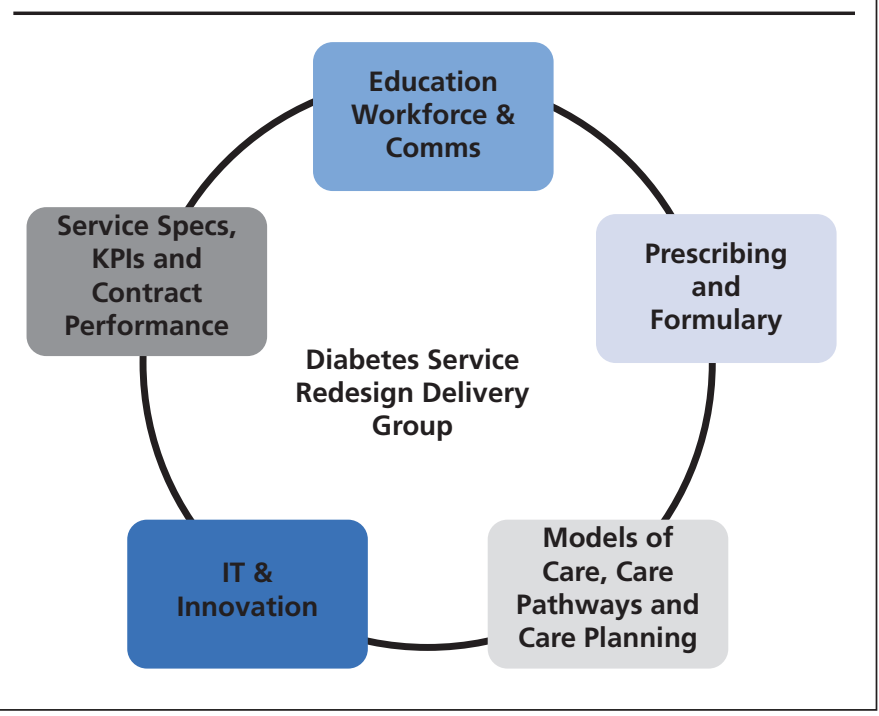

diabetologist) met every 2-3 weeks in the initial months to ensure momentum was maintained.

\section{Patients' views}

The patients and Diabetes UK representative involved in the work streams and the steering group were crucial when exploring how changes would be received by our service users. We were particularly struck by how much they valued being a part of a team that was helping create a service and a process that would engineer savings for the NHS. One member described it as his chance to give something back to the system that he has always felt he only took from. Furthermore, they were particularly happy that they could see that this saving was going directly into care for people with diabetes.

\section{Website and YourDiabetes brand development}

We did not previously have a strong web presence. Tentative work had begun to develop the hospital diabetes site so patients could easily access information. It was discussed and agreed that, rather than have two separate sites, we would join forces and develop a single diabetes website to represent both the CCG and trust. This would ensure that people with diabetes in the Vale of York would receive advice and similar access wherever they were receiving their diabetes care.

The HDT offered to pay the website set up cost of about f5000 from existing charitable funds. The site will be hosted as a micro-site within the existing hospital site to reduce running cost but will be accessible directly and have the appearance of a unique website. When live, the site will be administered by the CDT staff but will have equal representation of both the hospital and CCG identity.

The process of website development led to the creation of a brand name for the whole service - YourDiabetes (Figure 3), which will appear on all levels of the service to help break down traditional barriers and create a seamless service for the patients. 
Figure 3. New logo and brand

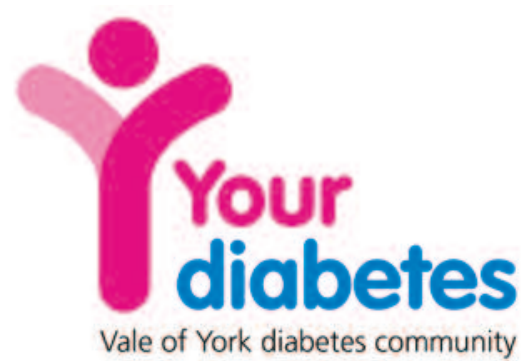

\section{Service specification and CCG business case}

The CCG project manager and other core members started drafting the service specification early in the process. This allowed it to be populated as the various subgroups reported developments. We initially reviewed the service specification and key performance indicators used by similar projects such as the Portsmouth service. This approach was helpful in providing a benchmark and framework, but we found it easier to create our own bespoke service specification. Shortly after we completed this work, NHS England circulated a draft of the recommended diabetes specification. This was affirming as we were able to benchmark against it and were confident we had attained and possibly improved on the required standard.

The service specification and the model of care documents were key documents in the business case submission to the CCG governing body. The case outlined the historical context, local developments, case for change, the new model promoting integrated community based care and stakeholder involvement. The financial modelling presented was fundamental to the business case, as savings were clearly demonstrated along with why and how this should be re-invested into diabetes care in the locality. Patient involvement and support for this process were critical and prominently highlighted.

The business case was approved by the CCG governing body without any changes or requests for additional information. There was universal agreement that this process of commissioning should be an exemplar and used when other service developments were being considered by VoY-CCG.

\section{The next steps}

In keeping with best practice commissioning, VoY-CCG are looking to risk share by identifying clear and measurable outcomes that attach to the financial implications of the care model. As YTHFT already provide the specialist diabetes service, the contracting teams from the two organisations are currently discussing a contract variation to allow for all the financial assumptions to be reflected. This in hindsight could have been agreed in principle at an earlier stage of the development process to prevent hold ups in the roll out.

\section{Conclusion}

This journey has been a steep learning curve and challenging.

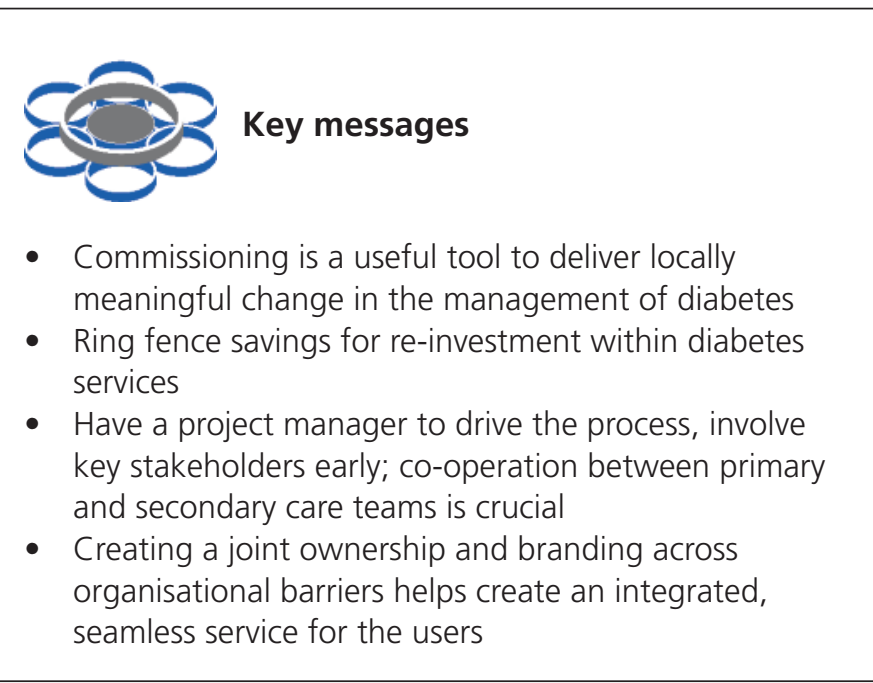

For most of us this kind of large scale service change is outwith our usual roles. It has however been hugely rewarding to be a part of a fundamental process of change that we hope will transform diabetes care provision in our locality. We hope that the telling of this story will help and encourage others in the diabetes community who are considering going down a similar path.

Conflict of interest None.

Acknowledgment We would like to thank Janine Reid, our initial project lead, for providing the impetus and co-ordination that set this project in motion.

\section{References}

1. Hex N, Bartlett C, Wright D et al. Estimating the current and future costs of type 1 and type 2 diabetes in the UK, including direct health costs and indirect societal and productivity costs. Diabet Med 2012;29:85562. http://dx.doi.org/10.1111/j.1464-5491.2012.03698.x

2. NHS Diabetes. Commissioning excellent diabetes care: an at a glance guide to the NHS Diabetes commissioning resource.2nd edn. February 2012. http://www.diabetes.org.uk/Documents/nhs-diabetes/commissioning/commissioning-at-a-glance-guide-2nd-edition.pdf

3. Diabetes UK. State of the Nation. England. 2013 http://www.diabetes.org.uk/Documents/About\%20Us/What\%20we\%2 Osay/0160b-state-nation-2013-england-1213.pdf (Accessed May 2014).

4. National Audit Office The management of adult diabetes services in the NHS. -. May 2012. http://www.nao.org.uk/wp-content/uploads/2012/ 05/121321.pdf (Accessed May 2014).

5. Goenka N, Turner B, Vora J et al. Commissioning specialist diabetes services for adults with diabetes: summary of a Diabetes UK Task and Finish group report. Diabet Med 2011;28:1494-500. http://dx.doi.org/10.1111/j.1464-5491.2011.03410.x

6. NHS Diabetes. Best practice for commissioning diabetes service: An integrated care framework. March 2013. https://www. diabetes.org.uk/ Documents/Position\%20statements/best-practice-commissioning-diabetes-services-integrated-framework-0313.pdf (Accessed May 2014).

7. Kar P. The 'super six' for the acute trust, all else under primary care? Pract Diabetes 2011;28:308-09. http://dx. doi.org/10.1002/pdi.1622

8. Rea RD, Gregory S, Browne $\mathrm{M}$ et al. Integrated diabetes care in Derby: New NHS organisations for new NHS challenges. Pract Diabetes 2011;28:312-13. http://dx.doi.org/10.1002/pdi.1624

9. NHS Diabetes. Commissioning diabetes - an integrated care framework March 2013. http://www.leicestershirediabetes.org.uk/816.html (Accessed May 2014) 